Original Paper http://ajol.info/index.php/ijbcs http://indexmedicus.afro.who.int

\title{
Effet de la densité de charge et de différents types de substrat sur la productivité du ver de fumier Eisenia foetida (Savigny, 1826) pour l'alimentation des sujets d'élevage
}

\author{
Pierre K. HOUNDONOUGBO ${ }^{1,2}$, Antoine CHIKOU ${ }^{1 *}$, Epiphane SODJINOU ${ }^{3}$, \\ Alphonse ADITE ${ }^{2}$, Clément BONOU ${ }^{4}$, Guy A. MENSAH ${ }^{5}$ et Philippe LALEYE ${ }^{1}$ \\ ${ }^{1}$ Université d'Abomey-Calavi, Faculté des Sciences Agronomiques, \\ Laboratoire d'Hydrobiologie et d'Aquaculture. 03 BP 2819 Jéricho, Cotonou. Bénin. \\ ${ }^{2}$ Université d'Abomey-Calavi, Faculté des Sciences et Techniques, \\ Unité de Recherche sur les Zones Humides 01 BP 526 Cotonou. Bénin. \\ ${ }^{3}$ Université des Sciences Arts et Techniques de Natitingou, Département d'Economie et de Sociologie Rurales, \\ École Nationale Supérieure des Sciences et Techniques Agronomiques de Djougou, B.P. 73 Djougou. \\ 4 Laboratoire de Recherche en Biologie Appliquée/ Ecole Polytechnique d'Abomey-Calavi/Université \\ d'Abomey-Calavi. 01 BP 2009, Cotonou, Bénin. \\ (5) Institut National des Recherches Agricoles du Bénin, Centre de Recherches Agricoles d'Agonkanmey.01 BP \\ 884. Recette Principale Cotonou 01, Bénin. \\ *Auteur correspondant ; E-mail : chikoua@yahoo.fr; Tél : +22996691353
}

\section{REMERCIEMENTS}

Ce travail a bénéficié de l'apport financier du Projet Aliment/Poisson qui est un volet du Programme de Productivité Agricole en Afrique de l'Ouest (PPAAO). Les auteurs remercient les responsables du programme à divers niveaux.

\section{RESUME}

La présente étude vise la mise au point de techniques efficaces pour la production intensive de ver de fumier. L'effet de la densité de charge et l'influence de la nature du substrat ont été étudiés sur les performances de production du ver du fumier Eisenia foetida durant 8 semaines. Les fumiers de bovin, d'ovin, de lapin et de porc sont utilisés à divers taux dans le substrat pour produire le ver de fumier à partir de deux densités différentes $\left(\mathrm{d} 10=10\right.$ vers $/ 5,2 \mathrm{~cm}^{3}$ et $\mathrm{d} 30=30$ vers $/ 5,2 \mathrm{~cm}^{3}$. Le substrat témoin est la terre de barre de la station d'élevage. Au total 90 bacs ont été utilisés suivant les traitements $75 \%, 50 \%$ et $25 \%$ du fumier concerné, le complément étant la terre de barre. Le dispositif expérimental est un bloc aléatoire complet constitué de 5 traitements et de 2 densités. L'analyse de variance à deux critères (ANOVA 2) a permis de comparer la productivité des substrats en fonction des densités. Les principaux résultats obtenus montrent que les substrats utilisés influencent différemment la productivité des vers de fumier de même que les densités de mise en culture de ceux-ci $(\mathrm{p}<0,05)$. La classification ascendante hiérarchisée indique que les substrats les plus productifs sont ceux de bovin $(137,38 \pm 38,00$ vers /semaine) et de lapin $(167,11 \pm 25,00$ vers/semaine $)$. La production des vers de fumier dans les petits agro-systèmes est donc possible et pourrait contribuer à la réduction des coûts d'alimentation des sujets d'élevage.

(C) 2017 International Formulae Group. All rights reserved.

Mots clés: Ver de fumier, substrats d'élevage, densité, productivité. 


\title{
Effect of loading density and different substrate types on the productivity of manure worm Eisenia foetida (Savigny, 1826) for the feeding of livestock
}

\begin{abstract}
The present study aims at the development of effective techniques for the intensive production of worm of manure. The effect of the density of load and the influence of nature of the substrate were studied on the performances of production of the worm of the manure $E$ foetida during 8 weeks. The manures of bovine, sheep, rabbit and pig are used at various rates in the substrate to produce the worm of manure starting from two different densities $\left(\mathrm{d} 10=10\right.$ worms by $5.2 \mathrm{~cm}^{3}$ and $\mathrm{d} 30=30$ worms by $5.2 \mathrm{~cm}^{3}$. The pilot substrate is the ground of bar of the station of breeding. In total 90 vats were used according to the treatments $75 \%, 50 \%$ and $25 \%$ of the manure concerned, the complement being ground of bar. The experimental device is a complete random block with 5 treatments with three repetitions by density. The variance analysis to two criteria (ANOVA 2) made it possible to compare the productivity of the substrates according to the two densities. The principal results obtained show that the substrates used differently influence the productivity of the worms of manure just as the densities of setting in culture of those $(p<0.05)$. Hierarchical ascending classification indicates that the most productive substrates are those of bovine $(137.38 \pm 38.00$ worms/week) and of rabbit $(167.11 \pm 25.00$ worms by week). The production of the worms of manure in the small agro-systems is thus possible and could contribute to the reduction of the costs of food of the breeding subjects.

(C) 2017 International Formulae Group. All rights reserved.
\end{abstract}

Keywords: Worm of manure, substrates of breeding, density, productivity.

\section{INTRODUCTION}

L'élevage des vertébrés sauvages de petite taille (oiseaux, reptiles, amphibiens, rongeurs et autres) et des invertébrés (annélides, escargots et autres mollusques), connu sous le nom de Mini-Elevage Non Conventionnel (MENC) est un moyen efficace de conciliation des objectifs de conservation de la nature et de la satisfaction des besoins alimentaires des populations (Mensah et al., 2001; Djossa et al., 2014; Chikou et al., 2016).

Selon Cakpo-Chichi (2012) et Sodjinou et al. (2016), plusieurs espèces animales font déjà l'objet du MENC. Parmi celles-ci, figurent en bonne place, les vers de terre, bien valorisés dans l'alimentation des animaux comme la volaille et les poissons (Sodjinou et al., 2016; Chikou et al., 2016). Leur importance dans la gestion de la fertilité des terres est également bien connue (Gérard, 2012).

Pour Vielma-Rondon (2003), les vers de terre sont très riches en protéine $(60-70 \%$ de protéine brute du poids sec) et contiennent de faibles quantités de fibre $(<5 \%$ du poids $\mathrm{sec})$.

Selon Garg et al. (2005), ils sont hautement concentrés en énergie digestible $(16-17 \mathrm{~kJ} / \mathrm{g})$ et constituent de ce fait un bon candidat pour l'alimentation des animaux d'élevage.

De plus la vermiculture permet de combiner plusieurs avantages dont la gestion de déchets organiques diversifiés et la production du lombricompost qui présente des niveaux de contamination en microorganismes pathogènes bien plus faibles que le compost conventionnel (Ndegwa, 2001).

L'élevage des vers de terre, en association avec l'achatiniculture a déjà donné des résultats encourageants (Zannou, 2000; Cakpo-Chichi, 2012; Djossa, 2014).

De plus les substrats composés des fumiers de lapin, petits ruminants et volaille ont été également testés avec des résultats intéressants (Aweha et Mensah, 2002; 
Edénakpo, 2007; Cakpo-Chichi, 2012; Djossa et al., 2014). Mais aucun de ces travaux n'a abordé la richesse en fumier du substrat, ni la densité de mise en charge des vers de fumier pour une production optimale. La présente étude a été initiée pour (1) mesurer la vitesse de production de biomasse des vers de terre (Eisenia foetida) sur des substrats enrichis avec le fumier, et (2) déterminer la densité optimale de production des vers de fumier.

\section{MATERIEL ET METHODES}

Origine des géniteurs de vers de terre ensemencés

Les géniteurs de E. foetida ensemencés proviennent $\mathrm{du}$ village Agolin, Lowé, commune d'Adjohoun (N 06 $39^{\prime} 378^{\prime \prime}$, E $\left.002^{\circ} 28^{\prime} 571^{\prime \prime}\right)$ dans la vallée de l'Ouémé au Bénin. La collecte des vers est manuelle et a consisté à une fouille dans les 10 premiers centimètres de la vase suite à l'observation des territicules rejetés par ces derniers à la surface des litières. Les vers collectés sont conservés avec une partie de leur substrat d'origine et transférés au laboratoire à l'aide des matières plastiques munies de perforations pour faciliter leur aération. La mise en culture a été réalisée 2 jours après le transfert des vers en station au Laboratoire d'Hydrobiologie et d'Aquaculture de la Faculté des Sciences Agronomiques de l'Université d'AbomeyCalavi. Pour ce faire, les vers de fumier ont été nettoyés et pesés ensemble et le poids moyen déterminé à l'aide de la balance de marque "DIGITAL SCAL" de capacité $500 \mathrm{~g}$ et de précision $0,01 \mathrm{~g}$. Les poids moyens des vers ensemencés sont respectivement de 0,39 $\pm 0,03 \mathrm{~g}$ et de $0,42 \pm 0,02 \mathrm{~g}$ pour les différentes densités.

\section{Densités de mise en culture}

Deux densités d'élevage ont été utilisées. La densité d10 correspond à 10 vers /bac d'élevage soit $1.933 \mathrm{vers} / \mathrm{m}^{3}$ de substrat pour une biomasse de $3,89 \pm 0,04 \mathrm{~g}$ et la densité $\mathrm{d} 30$ correspondant à 30 vers/ bac soit $5.798 \mathrm{vers} / \mathrm{m}^{3}$ de substrat pour une biomasse de $12,6 \pm 0,02 \mathrm{~g}$.

\section{Substrats d'élevage et leur composition}

Quatre substrats ont été utilisés à savoir le fumier de bovin, le fumier de porc, le fumier de lapin et le fumier d'ovin. Le substrat témoin est constitué de terre de barre de la station d'élevage. Pour chaque substrat, trois niveaux de traitement ont été adoptés: $25 \%$ de fumier $+75 \%$ de terre de barre, $50 \%$ de fumier $+50 \%$ de terre de barre et $75 \%$ de fumier $+25 \%$ de terre de barre.

$\mathrm{Au}$ préalable les fumiers sont désinfectés par un léger chauffage à feu doux à l'aide d'une marmite de $100 \mathrm{~L}$ puis arrosés chaque jour pendant une semaine afin de les mouiller complètement mais aussi et surtout pour dégager toute la chaleur due au début de la décomposition des matières organiques souvent observées dans ces types de fumiers, et qui sont des conditions nécessaires pour une culture réussie des vers de terre (CakpoChichi, 2012 ; Djossa et al., 2014). La terre de barre utilisée comme substrat témoin présente les caractéristiques physiques et chimiques suivantes: $15,3 \%$ d'Argile; 5,4\% de limon; $77,3 \%$ de sable et $0,05 \%$ d'Azote total. Elle a une teneur en matière organique de $2,64 \%$; la somme totale des bases échangeables de 2,44 még/ $100 \mathrm{~g}$ et une capacité d'échange cationique de 4,4. (Azontondé, 1992).

\section{Dispositif expérimental}

Le dispositif expérimental a été un bloc aléatoire complet comportant les cinq substrats dont le témoin et les deux densités. Chaque substrat contient trois niveaux de traitement correspondant à sa concentration en fumier $(25 \%, 50 \%$ et $75 \%)$. Les vermissières ont été installées selon la méthode de Djossa (2014). Elles ont été disposées à l'ombre et fermées par une toile moustiquaire à mailles très fines pour limiter les risques d'attaque des 
prédateurs. Le dispositif est couvert de claies pour diminuer l'effet de la lumière et l'infiltration directe de l'eau de pluie parfois acide.

Au total 90 bacs uniformes de $45,0 \mathrm{~cm}$ de longueur, de 23,0 cm de largeur et de 21,3 $\mathrm{cm}$ de hauteur ont été utilisés pour les cinq substrats d'élevage à trois niveaux de traitement et les deux densités. La Figure 1 récapitule les différents niveaux de concentration des fumiers dans le substrat. Ce dispositif a été répété 3 fois.

\section{Ration et fréquence de nourrissage}

Une ration journalière correspondante à $50 \%$ de la biomasse, composée des débris de cartons et des papiers journaux, leur a été servie une fois par semaine et ceci juste après les contrôles de croissance. Cette consommation moyenne équivalente à la moitié de leur poids est retenue du fait que le substrat en lui même constitue déjà une première source de nourriture pour les vers (Hardouin, 2001).

\section{Contrôle de croissance numérique et pondérale}

La récolte a consisté à un tri à la main ou un ramassage manuel des vers. Le bac d'élevage contenant les vers est déversé sur une table éclairée par une source de lumière. Les vers fuyant la lumière, s'enfouissent rapidement sous le substrat. Le compost par couches est ensuite enlevé jusqu'à ce qu'il n'y ait plus rien sur la table hormis un amas de vers sous une mince couche de compost. Les vers ont été ensuite prélevés, comptés et pesés par traitement.
Contrôle des paramètres du milieu
d'élevage
$\mathrm{La}$ température et le $\mathrm{pH}$ au cœur $\mathrm{du}$ substrat ont été mesurés tôt le matin à 7 heures une fois par semaine. Les mesures ont été prises à l'aide d'un oxythermomètre WTW Oxi 197. Le pH a été mesuré à 0,1 unité de $\mathrm{pH}$ près à l'aide d'un pH-mètre PIERRON HI 1290.

\section{Collecte de données biométriques \\ Les données biométriques ont été} collectées par semaine. Au début de l'expérience, les vers ont été acclimatés pendant une semaine leur permettant de s'adapter aux nouvelles conditions écologiques. L'augmentation du nombre de vers de fumier a été notée ainsi que le poids total de vers par bac. L'essai a duré 8 semaines.

\section{Analyse statistique}

Les données collectées ont été analysées au moyen des logiciels StatView 5.1 et $\mathrm{R}$. Des courbes de variation du nombre de vers ont été tracées et des moyennes de biomasse sont calculées. Le logiciel StatView 5.1 a permis de réaliser les analyses de la variance à un et à deux facteurs (Anova 1 et 2) afin de comparer les productivités des substrats et la densité optimale de production des vers de terre. Au préalable, les conditions de normalité et d'égalité des variances ont été vérifiées au moyen des tests Ryan Joiner et de Bartlett's (Mensah et al., 2001). Le test de LSD (Least Significant Difference) de Fisher et celui de Student Newman Keul ont été utilisés pour comparer deux à deux les moyennes des productivités au niveau des différents substrats. Le logiciel $\mathrm{R}$ a permis de réaliser une classification ascendante hiérarchisée des substrats d'élevage. 


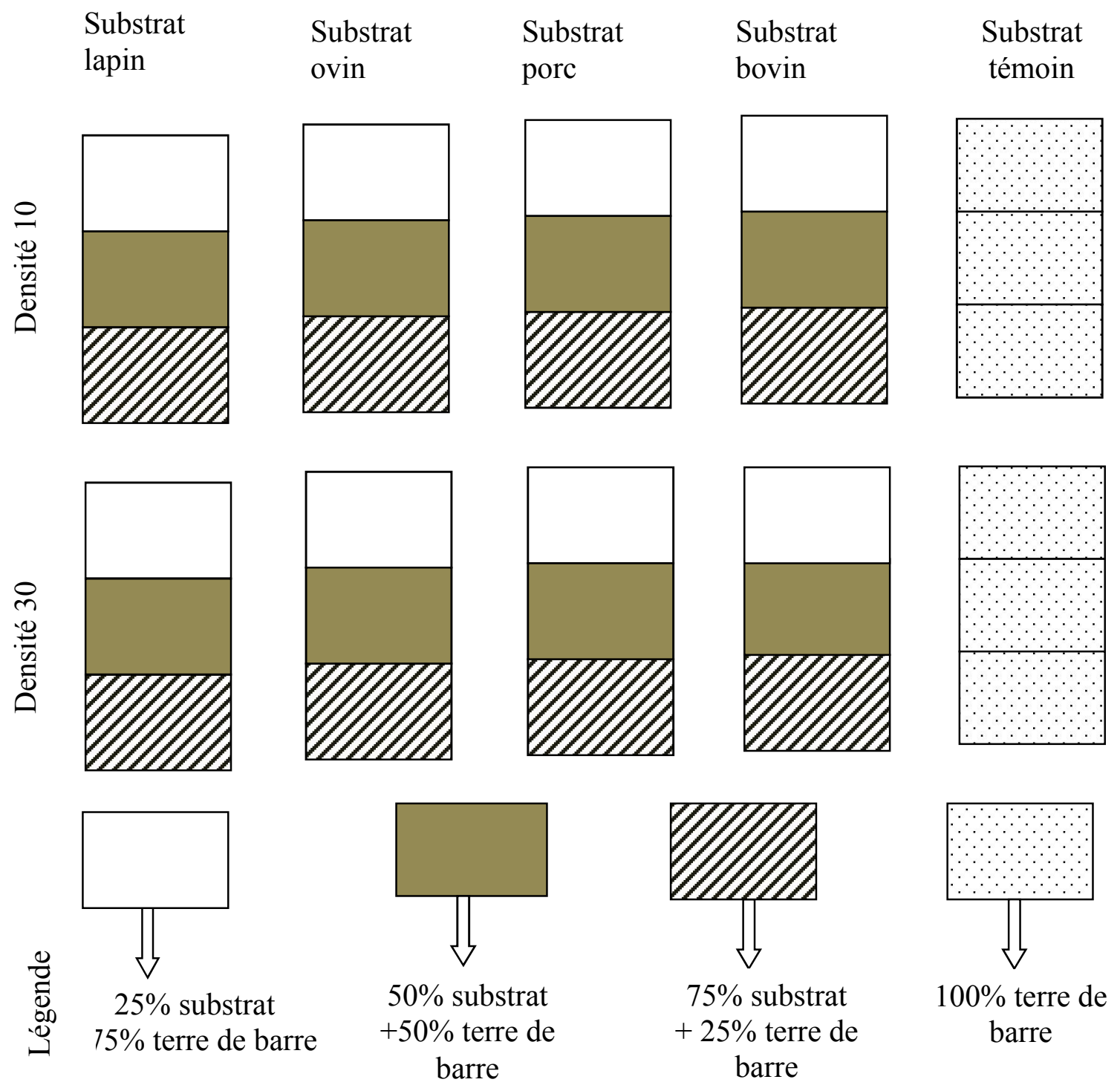

Figure 1: Dispositif expérimental d'élevage des vers de fumier (Eisenia foetida) contenant différents substrats.

\section{RESULTATS}

Qualité du milieu de culture

Les valeurs moyennes de la température ont varié de $24 \pm 0,5^{\circ} \mathrm{C}$ à $25 \pm$ $0,7^{\circ} \mathrm{C}$, celles du pH de 7,75 $\pm 0,30$ à $7,65 \pm$ 0,40 . Aucune différence significative n'existe entre les traitements pour ces différents paramètres $(\mathrm{P}>0,05)$.
Effet de la densité et de la nature du substrat sur la croissance numérique et pondérale des vers de fumier

La Figure 2 indique le nombre de ver de fumier et leur biomasse respectivement à densité $\mathrm{d} 10$ et $\mathrm{d} 30$. 
Une tendance évolutive croissante $d u$ nombre de ver ainsi que la biomasse, avec une différence hautement significative $(\mathrm{P}<0,05)$, est observée pour tous les traitements par rapport au témoin en considérant les densités de mise en charge.

Le nombre des vers de fumier récoltés et leur biomasse ont été plus élevés pour la densité d10 (14140 vers et 4667,95 g). La nature du substrat a significativement impacté la productivité des vers de fumier $(\mathrm{P}<0,05)$. Ainsi le substrat lapin (9024 vers pour $2839,59 \mathrm{~g})$ et le substrat bovin (7419 vers pour $2473,16 \mathrm{~g}$ ) ont présenté de meilleures performances de production des vers.

Effet du taux de fumier sur la croissance numérique et pondérale des vers de fumier.

La Figure 3 montre la production numérique et pondérale du ver de fumier en fonction de la concentration du fumier dans le substrat.

La concentration du substrat en fumier a été très déterminante pour la productivité des vers. Le nombre de vers récoltés ainsi que la biomasse sont proportionnels à la richesse du substrat en fumier. Ainsi, les substrats renfermant $75 \%$ de fumier ont été les plus productifs. La probabilité associée au test de Fisher $(\mathrm{P}=0,0001)$ indique une différence significative entre le nombre de ver et leur biomasse pour ces différents traitements.

Evolution du poids moyen des vers de fumier en fonction des semaines

Les Figures 4 et 5 montrent l'évolution en poids des vers de fumier (E. foetida) au cours de l'essai respectivement à densité d10 et $\mathrm{d} 30$.

$\mathrm{Au}$ début de l'essai, le poids moyen des vers était de $0,390 \pm 0,030 \mathrm{~g}$ et $0,420 \pm$ $0,020 \mathrm{~g}$ respectivement à densité $\mathrm{d} 10$ et $\mathrm{d} 30$.

En fin d'expérience, il est passé à $2,569 \pm 0,020 \mathrm{~g}$ et $1,880 \pm 0,010 \mathrm{~g}$ pour le lapin respectivement à $\mathrm{d} 10$ et $\mathrm{d} 30$, à $2,386 \pm$
$0,030 \mathrm{~g}$ et $1,578 \pm 0,010 \mathrm{~g}$ pour le bovin respectivement à $\mathrm{d} 10$ et $\mathrm{d} 30$. Quant aux autres substrats, les poids moyens sont passés à $0,949 \pm 0,040 \mathrm{~g}$ et $2,052 \pm 0,020 \mathrm{~g}$ pour le ovin respectivement à $\mathrm{d} 10$ et $\mathrm{d} 30$, à $0,887 \pm$ $0,010 \mathrm{~g}$ et $2,172 \pm 0,030 \mathrm{~g}$ pour le porc respectivement à d10 et $\mathrm{d} 30$. Le gain de poids moyen le plus élevé est de $0,018 \mathrm{~kg}$ de vers/ $\mathrm{m}^{3}$.

Les traitements ont influencé différemment l'évolution en poids des vers de fumier. Les meilleures croissances en poids ont été obtenues avec la densité d10 pour les traitements en fumier de lapin et de bovin.

Les croissances les plus faibles ont été obtenues avec les fumiers ovins et porc pour la densité d10. Les vers de fumier de la terre de barre non enrichie ont perdu du poids. Le test d'Anova 1 effectué, a montré de différence significative $(\mathrm{P}<0,05)$ entre les traitements en fonction des semaines.

En effet, les substrats porc $(0,391 \mathrm{~g}$ à $2,171 \mathrm{~g})$ et ovin $(0,389 \mathrm{~g}$ à $2,051 \mathrm{~g})$ ont produit des vers de fumier de poids moyens respectivement plus élevés que ceux produits dans les substrats lapin $(0,381$ à $1,880 \mathrm{~g})$ et bovin $(0,341 \mathrm{~g}$ à $1,578 \mathrm{~g})$ avec une différence significative $(\mathrm{P}<0,05)$ à densité $\mathrm{d} 30$ en fonction des semaines.

Effet croisé de la densité et de la nature du substrat sur la croissance des vers de compost.

Les densités de mise en charge aussi bien que la nature du substrat ont influencé significativement la production des vers de fumier $(\mathrm{P}<0,05)$.

L'analyse de variance à deux critères (ANOVA 2) indique que l'effet croisé des deux paramètres est aussi significatif pour la production des vers de compost $(\mathrm{P}<0,001)$.

La classification ascendante hiérarchisée réalisée à partir des abondances numériques et pondérales a permis d'avoir 3 groupes de substrat à une distance 
d'agrégation de 30 (Figure 6) selon le niveau de production. Ces regroupements s'expliquent par une forte hétérogénéité aussi bien numérique que pondérale des vers de fumier en fin de culture (Figures 2 et 3 ).

Les traitements à base du fumier de bovin et du fumier de lapin de niveau de concentration élevé (75\%) ont présenté les meilleures productions numériques et pondérales (Figures 2 et 3 ). Ce qui est confirmé par la classification ascendante avec des groupes de substrat bien déterminés en fonction de la production. L'analyse de variance à deux facteurs indique qu'il existe une différence hautement significative des productions de chaque groupe de substrat $(\mathrm{P}<$ $0,05)$.

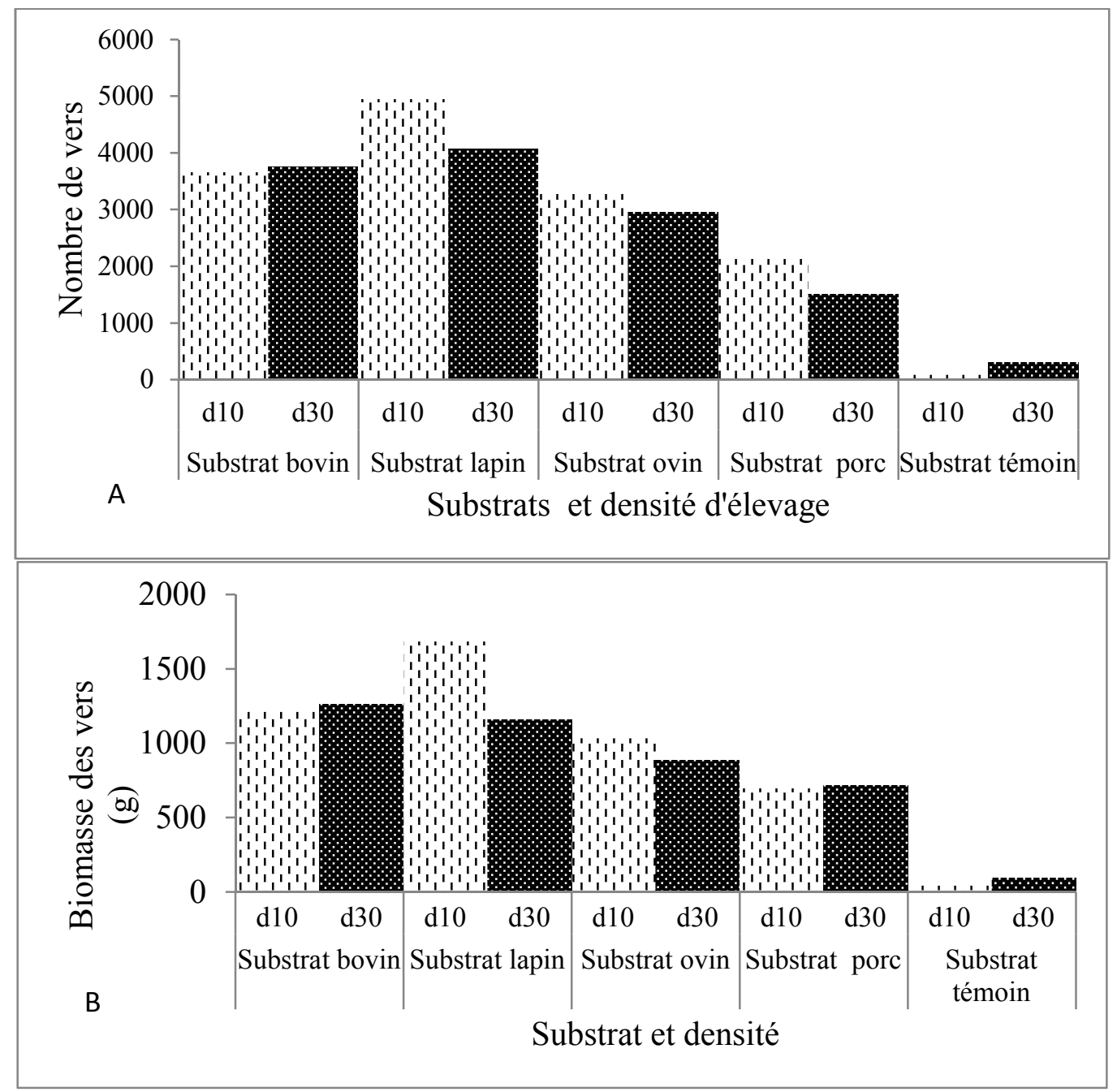

Figure 2: Influence de la densité et de la nature du substrat. (A): influence sur la production numérique et $(\mathrm{B})$ : influence sur la biomasse des vers de compost (E. foetida). 


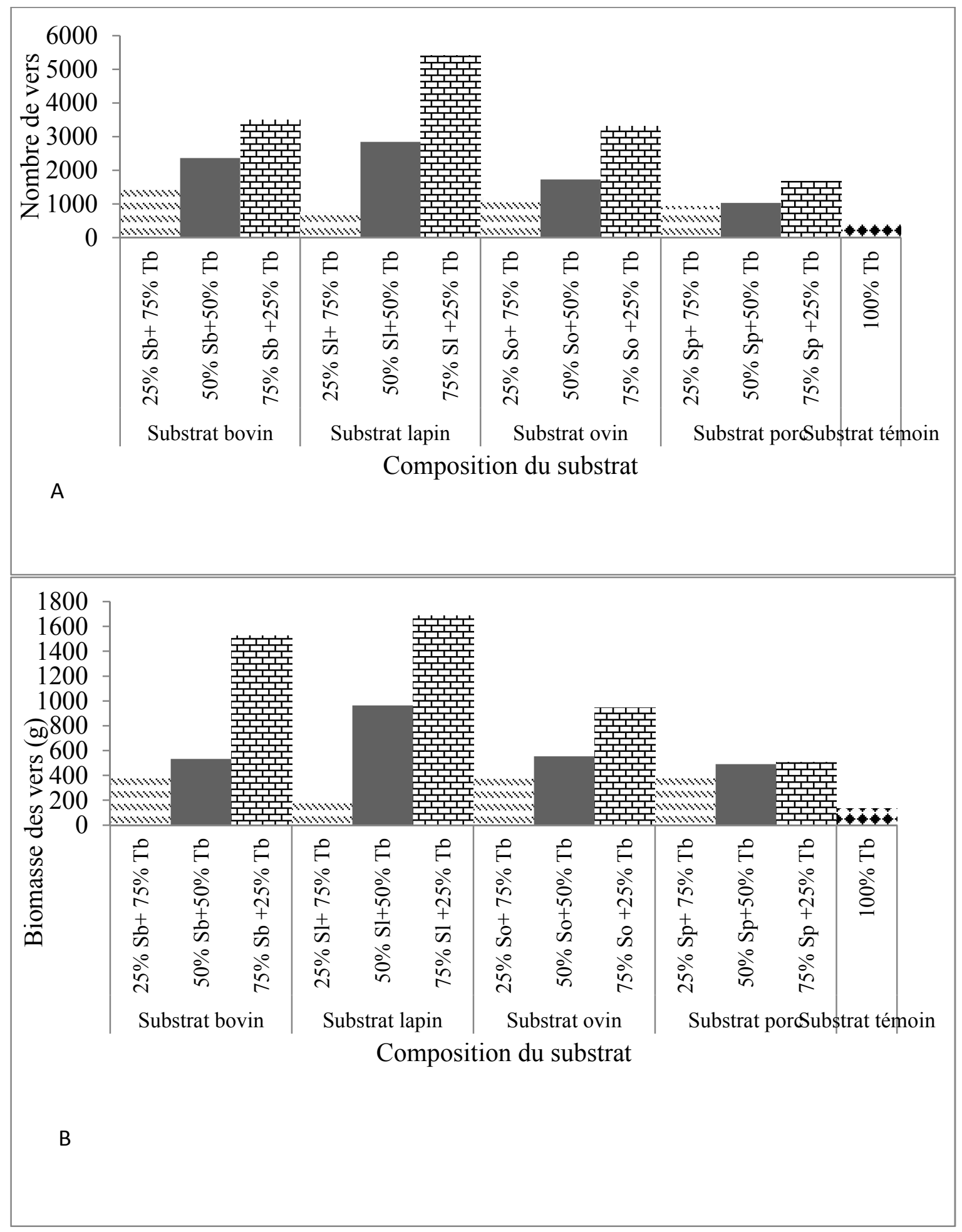

Figure 3 : Influence de la concentration du substrat. (A) : influence sur la productivité numérique et (B) : influence sur la biomasse des vers de compost. $\mathrm{Sb}=$ substrat bovin ; $\mathrm{Tb}=$ terre de barre; $\mathrm{Sp}=$ substrat porc; $\mathrm{So}=$ substrat ovin et $\mathrm{Sl}=$ Substrat lapin. 


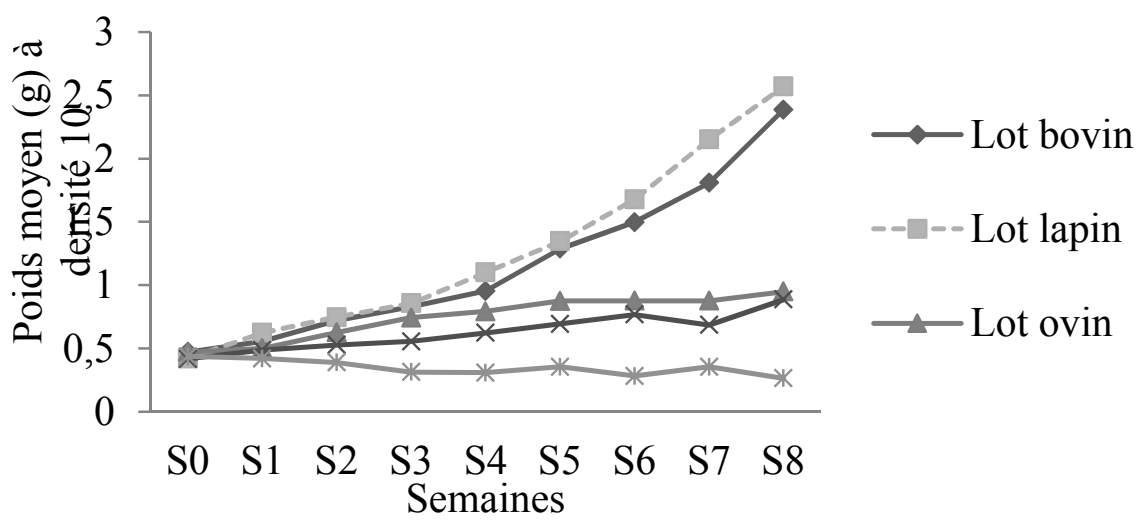

Figure 4: Croissance en poids des vers de fumier (E. foetida) au cours de l'essai à densité d10.

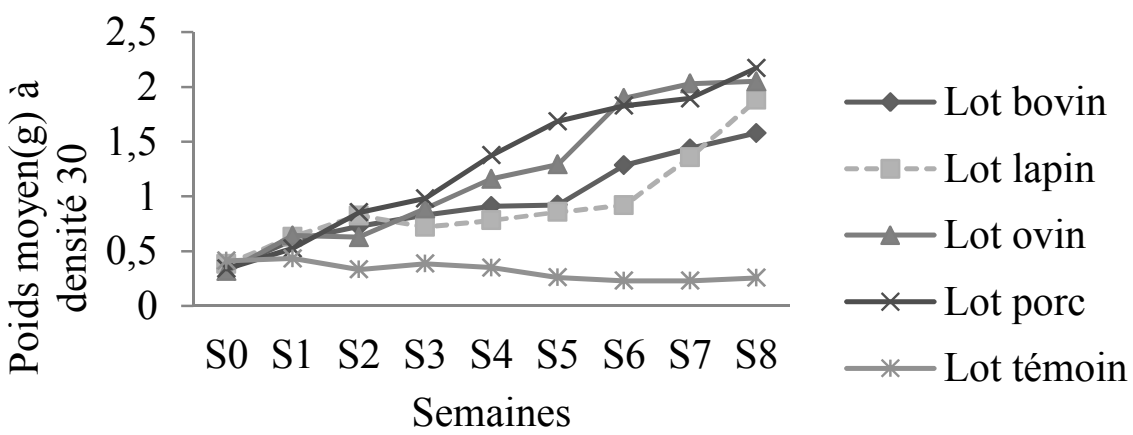

Figure 5: Croissance en poids des vers de fumier (E. foetida) au cours de l'essai à densité d30.

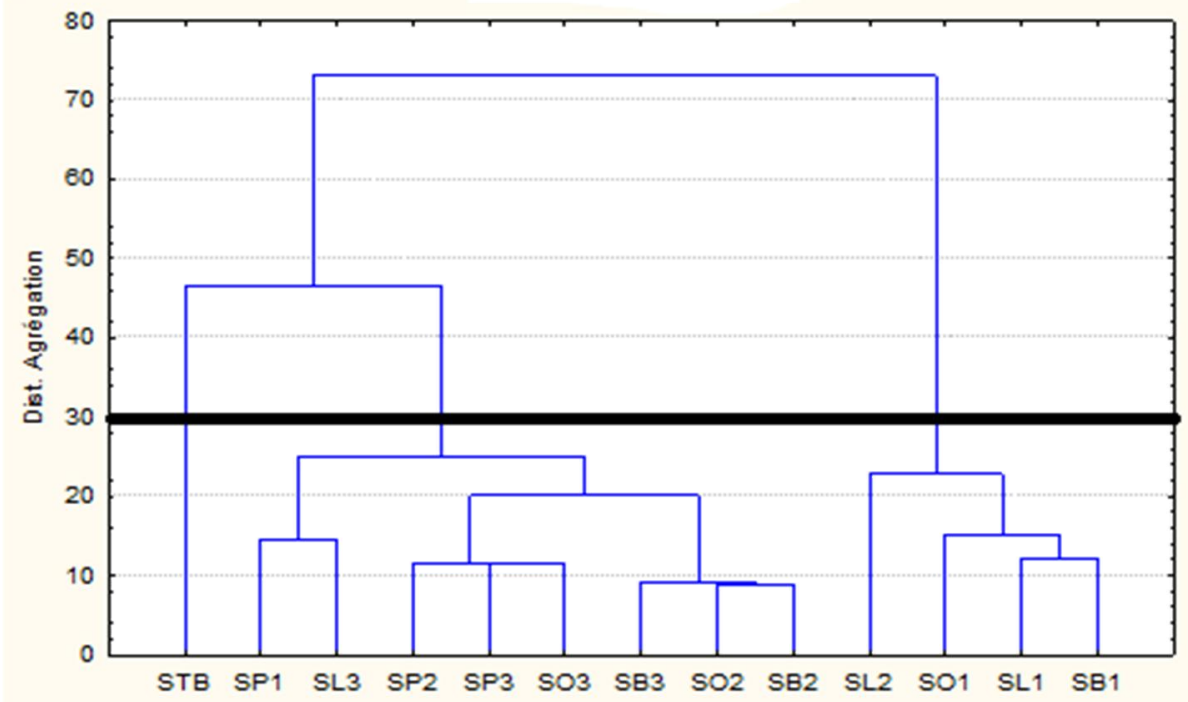

Figure 6: Classification ascendante hiérarchisée des traitements en fonction de la productivité. (SL1 $=75 \% \mathrm{Cl}+25 \% \mathrm{~Tb} ; \quad \mathrm{SB} 1=75 \% \mathrm{Db}+25 \% \mathrm{~Tb} ; \quad \mathrm{SO} 1=75 \% \mathrm{Co}+25 \% \mathrm{~Tb} ; \quad \mathrm{SL} 2=50 \% \mathrm{Cl}+50 \% \mathrm{~Tb} ; \quad \mathrm{SL} 3=25 \% \mathrm{Cl}+75 \% \mathrm{~Tb}$; $\mathrm{SP} 1=75 \% \mathrm{Dp}+25 \% \mathrm{~Tb} ; \mathrm{SB} 3=25 \% \mathrm{Db}+50 \% \mathrm{~Tb} ; \mathrm{SB} 2=50 \% \mathrm{Db}+50 \% \mathrm{~Tb} ; \mathrm{SO} 2=50 \% \mathrm{Co}+50 \% \mathrm{~Tb} ; \quad \mathrm{SO} 3=25 \% \mathrm{Co}+75 \% \quad \mathrm{~Tb}$; $\mathrm{SP} 2=50 \% \mathrm{Dp}+50 \% \mathrm{~Tb} ; \mathrm{SP} 3=25 \% \mathrm{Dp}+75 \% \mathrm{~Tb} ; \mathrm{STB}=100 \% \mathrm{de} \mathrm{Tb}$. 


\section{DISCUSSION}

Les températures relativement élevées obtenues les premières semaines sont dues à la poursuite de la décomposition qui n'est pas terminée pendant le pré compostage. Ces valeurs sont dans les normes indiquées par Hardouin (2001) pour la lombriculture et ne constituent pas un facteur limitant pour la production des vers de fumier.

Les différentes densités de mise en culture ont influencé significativement la productivité des vers de fumier dans les substrats d'élevage. Cette influence a été aussi signalée par El-Sayed (2002), Ma et al. (2006), Schram et al. (2006), Wirat (2011) et Chattopadhyay et al. (2013) sur des sujets d'élevage. L'abondance numérique des vers de fumier obtenus pour la densité de 1933 vers $/ \mathrm{m}^{3}$ est meilleure à celle obtenue pour la densité de 5.798 vers $/ \mathrm{m}^{3}$ qui est parfaitement en phase avec les densités de 5.689 vers $/ \mathrm{m}^{3}$ utilisées respectivement par Cakpo-Chichi (2012) et Djossa et al. (2014). La production plus élevée des vers de fumier à densité 10 est due à la disponibilité de l'espace et/ou de l'aliment limitant ainsi la compétition intraspécifique.

Du point de vue de la nature du substrat, les résultats, en ce qui concerne l'abondance numérique, confirment ceux de Aweha et Mensah (2002) qui ont eu un peu plus tôt une meilleure production de vers de fumier sur un substrat à base de crottes de lapin. Djossa et al. (2014) ont également classé la bouse de vache comme étant le substrat le plus productif à travers un test de production de biomasse réalisé avec la bouse de vache, les crottes des ovins et des lapins.

Cependant, les résultats obtenus sont contraires à ceux de Garg et al. (2005) qui ont utilisé des crottes de caprin, d'ovin, de buffle, d'âne, de vache, de cheval et de chameau et qui ont classé les crottes de caprin et de buffle en tête. Les performances de production de biomasse ne sont donc pas liées à une espèce animale donnée mais plutôt à la «qualité » des déjections c'est-à-dire à la valeur nutritionnelle des restes de matières organiques que les vers de fumier peuvent valoriser (Djossa et al., 2014). Ainsi, la concentration du substrat en fumier ajoutée à l'effet de la composition bromatologique des fourrages appétés par les animaux, qui varient d'une saison à une autre et d'un milieu à un autre, justifient les diverses performances de production des vers de fumier obtenues.

Dans des conditions expérimentales similaires, Djossa et al. (2014) ont obtenu 0, $017 \mathrm{~kg}$ au bout de 7 semaines.

Pour l'ensemble de la présente étude, le poids corporel maximal des vers $(0,018 \mathrm{~kg})$ atteint au bout de 8 semaines d'élevage permet de tirer des conclusions valides pour la production massive des vers de fumiers en vue de l'alimentation des animaux domestiques.

\section{Conclusion}

La lombriculture est une technologie peu coûteuse qui peut être utilisée avec succès en milieu rural. L'efficacité de la production des vers de fumier dépend de la richesse des matières organiques constituant le substrat d'élevage et de la densité de mise en culture. Un substrat hautement concentré en fumier, confère au ver du compost un biotope propice à sa production.

\section{CONTRIBUTIONS DES AUTEURS}

PKH est l'investigateur principal. Il a effectué le suivi rigoureux de l'expérience et a collecté les données biométriques et physicochimiques. $\mathrm{AC}$ a défini et supervisé le protocole de collecte et d'analyse des données physico-chimiques. ES a participé au financement de l'étude. AA a participé à la finalisation du protocole d'étude ainsi que l'analyse des variables morphométriques. 
CB a participé à la finalisation du protocole en proposant les types de substrat utilisés pour l'élevage des vers. GAM a défini les méthodes appropriées d'analyses statistiques des données. PL a offert le cadre physique de travail et les facilitations nécessaires à la conduite des essais.

\section{REFERENCES}

Aweha F, Mensah A. 2002. Gestion des fumiers d'animaux herbivores d'élevage par compostage et lombricompostage. In : Actes de l'Atelier Scientifique 2. INRAB, Programme Régional SudCentre Bénin, Recherche Agricole pour le Développement; 155171.

Azontondé HA. 1992. Dégradation et Restauration des Terres de Barre au Sud Bénin. Centre National d'Agro-Pédologie (CENAP) : Cotonou, Bénin ; 23.

Cakpo-Chichi B. 2012. Association Achatiniculture et Vermiculture. Mémoire de Licence Professionnelle ENSTA/UAK, Bénin. 42p.

Chattopadhyay DN, Mohapatra BC, Adhikari S, Pani KC, Jena JK, Eknath AE. 2013. Effects of stocking density of Labeo rohita on survival, growth and production in cages. Aquacult. Int., 21(1): 19-29. DOI: 10.1007/s10499-0129528-2.

Chikou A, Sodjinou E, d'Almeida A, Mensah A, Houndonougbo P, Adjilé A, Mongbo R, AHounenou J, Houssou P, Adégbola P. 2016. Comment produire à coût réduit des aliments à base de vers de terre destinés à nourrir les poissons d'élevage au Bénin? Référentiel technicoéconomique, Grâce Divine Corporation, Porto-Novo, 44.

Djossa A, Alissou K, Mensah A, Sinsin A. 2014. Performance de production de biomasse du ver de fumier Eisenia foetida sur différents Substrats. Bulletin de la Recherche Agronomique du Bénin (BRAB) : 1840 : 26-31.

Edénakpo A. 2007. Diversité des lombrics dans les écosystèmes de la commune de Djidja au centre du Bénin et contribution de la lombriculture dans la gestion biologique des déchets organiques. Thèse de DESS, Université d'AbomeyCalavi, 76 p.

El-sayed AFM. 2002. Effect of stocking density and feeding levels on growth and feed efficiency of Nile tilapia Oreochromis niloticus. Aquacult. Res., 33: $621-626$.

Garg K, Chand A, Chhillar A, Yadav. 2005. Growth and reproduction of Eisenia foetida in various animal wastes during vermicomposting. Applied Ecology and Environmental Research, 3(2): 51-59.

Gérard G. 2012. Matières organiques Fiche $\mathrm{N}^{\circ} 4$, CRA PACA - Maison des Agriculteurs - 22, 4p.

Hardouin J. 2001. Guide technique d'élevage n06 sur le ver de terreau Perionyx INRAB, République du Bénin, 11p.

Ma A, Chen C, Lei J, Chen S, Zhuang Z, Wang Y. 2006. Turbot Scophthalmus maximus: stocking density on growth, pigmentation and feed conversion. Chin. J. Ocean. Limno., 24 (3) : 307 - 312.

Mensah A, Ekue M, Chadare J, Zannou A. 2001. Guide de formation en achatiniculture. Rapport multigraphié /LRZVH/CRA-Agonkanmey / INRAB / République du Bénin. $11 \mathrm{p}$.

Ndegwa PM, Thompson SA. 2001. Integrating composting and vermicomposting in the treatment and bioconversion of biosolids. Biores. Technol .,7(6): 107-112.

Schram E, Van der Heul JW, Kamstra A, Verdergem MCJ. 2006. Stocking density dependent growth of dover (Solea solea). Aquaculture, 252: 239 - 247. 
Sodjinou E, d'Almeida A, Mensah G, Hessavi P, Adjilé A, Chikou A, AHounénou J, Mongbo R, Houssou P, Adégbola P. 2016. Aliment, Ressource Alimentaire et Pratiques de Nourrissage dans les Exploitations Piscicoles du Bénin. Institut National des Recherches d Agricoles du Bénin : Porto-Novo ; 50p.

Vielma-Rondon R, Ovalles-Duran J.F, LeonLeal A, Medina A. 2003. Nutritional value of earth worm flour (Eisenia foetida) as a source of aminoacids and its quantitative estimation throughreversed phase Chromatography (HPLC) and precolumn derivation with o-phthal aldehyde (OPA). Ars Pharmaceutica, 44(1):43-58.

Wirat J. 2011. The effect of stocking density on yield, growth, and survival of Asian river catfish (Pangasius Nbocourti Sauvage, 1880) cultured in cages. Aquacult. Int., 19: 987-997.

Zannou A. 2000. Influence de l'alimentation sur la reproduction et le développement corporel d'Archachatina marginata élevé en association ou non avec des vers de terre. Mémoire de Diplôme d'Ingénieur de Technicien, Université d'Abomey-Calavi, 63p. 